\title{
Temporal decoupling of oxy- and deoxy-hemoglobin hemodynamic responses detected by functional near-infrared spectroscopy (fNIRS)
}

\author{
Nicoladie D. Tam ${ }^{\mathrm{a}}$, and George Zouridakis ${ }^{\mathrm{b}}$ \\ ${ }^{a}$ Department of Biological Sciences, University of North Texas, Denton, TX 76023, USA \\ ${ }^{b}$ Departments of Engineering Technology, Computer Science, and Electrical \& Computer \\ Engineering, University of Houston, Houston, TX 77204, USA; \\ nicoladie.tam@unt.edu, zouridakis@uh.edu
}

\begin{abstract}
This study provides experimental evidence that there is temporal decoupling between the hemodynamic responses of oxy- and deoxy-hemoglobin $(\mathrm{Hb})$ as detected by functional nearinfrared spectroscopy (fNIRS). Using 64 spatially distributed optrodes to record motor cortical activities during a free arm movement task (right-left and front-back movements), we detected that the temporal profile of oxy- and deoxy- $\mathrm{Hb}$ responses are desynchronized and decoupled (i.e., oxy- and deoxy- $\mathrm{Hb}$ levels do not rise and fall at the same time). We correlated four different measures of hemodynamic profiles with the arm movements, namely, oxy- $\left(\mathrm{HbO}_{2}\right)$ and deoxy-hemoglobin $(\mathrm{Hb})$ and their summation $\left(\mathrm{HbO}_{2}+\mathrm{Hb}\right)$ and difference $\left(\mathrm{HbO}_{2}-\mathrm{Hb}\right)$ signals. These measures correspond to the changes in oxygen delivery, oxygen extraction, total blood volume delivered, and total oxygenation with specific movement directions, respectively. They revealed different components of the hemodynamic response in a localized neuronal population in the motor cortex. The results suggested that, by using these four measures, oxygen delivery and oxygen extraction can be coupled in one movement direction, but decoupled in another movement direction for the same human subject executing the same movement task. Oxygen delivery and oxygen extraction do not always co-vary together temporally. Thus, using a single measure of oxygen delivery or extraction alone may not be sufficient to determine whether the cortical area is activated or deactivated. Rather, a combination of all four measures of hemodynamic signals that represent temporal coupling and decoupling of oxygen delivery and extraction is needed to differentiate the temporal profiles of neural activation and deactivation. It demonstrated that different hemodynamic measures can reveal temporally decoupled activation/deactivation patterns differentially during the right-left and front-back motor task. Therefore, relying on a single measure of deoxy-Hb may be insufficient to characterize the neural responses without the oxy- $\mathrm{Hb}$ measure. Orthogonal arm
\end{abstract}


Nicoladie D. Tam and George Zouridakis; Temporal decoupling of oxy- and deoxy-hemoglobin hemodynamic responses detected by functional near-infrared spectroscopy (fNIRS), Journal of Biomedical Engineering and Medical Imaging, Volume 1, Issue 2 (2014), PP 18-28

movement (right-left vs. front-back) directions can be differentiated based on the differential temporally coupled and decoupled hemodynamics.

Keywords: Functional near-infrared spectroscopy, fNIRS, hemodynamics, motor control, neural activation, temporal coupling.

\section{INTRODUCTION}

This study describes how functional near-infrared spectroscopy (fNIRS) can be used to reveal oxy- and deoxy-hemoglobin $(\mathrm{Hb})$ hemodynamic responses can be decoupled temporally during a cognitive motor task. fNIRS has been used as a noninvasive optical imaging technique to detect neural activation according to the hemodynamic response of the imaged neural tissues [1-4]. Hemoglobin molecules is known to absorb light in the near-infrared (NIR) spectral region, with a characteristic different absorption spectra for oxy- $\mathrm{Hb}\left(\mathrm{HbO}_{2}\right)$ and deoxy- $\mathrm{Hb}(\mathrm{Hb})$ based on the molecular chemical bonds [4]. Using fNIRS, real-time detection of brain activation can be inferred from the metabolic activities of the underlying neural tissues. The hemodynamic response is deduced from the temporal profile of oxygen delivery and extraction in the neural tissues, which is dependent on the metabolic activity and oxygen consumption in real time. It is ideal to detect cortical activity since NIR light can penetrate tissues up a depth of $2 \mathrm{~cm}$ without significant loss of the optical signals. Using finite element simulation methods, the depth of recorded neural activation can be computed from the absorption variations [5]. Thus, localized cortical activity can be deduced from the hemodynamic profile in real time [1-3], but it is often assumed that oxygen delivery and oxygen extraction are coupled and co-varied together. This study will verify whether such oxy- and deoxy-Hb hemodynamic profiles are coupled or decoupled temporally during a cognitive motor task.

As a brief review, $\mathrm{HbO}_{2}$ and $\mathrm{Hb}$ levels are detected optically, and computed from the modified Beer-Lambert law [4] based on refracted light scattered by the tissue. fNIRS can detect not just $\mathrm{HbO}_{2}$ level but also $\mathrm{Hb}$ level, since the difference in spectral absorption between oxy- and deoxy- $\mathrm{Hb}$ molecules provides direct estimation of oxy- and deoxy-Hb levels. In contrast, functional magnetic resonance imaging ( $\mathrm{fMRI}$ ) detects primarily deoxy-Hb level, but not oxy-Hb level, because it is based on the blood oxygen level dependent (BOLD) activity. This is because deoxy- $\mathrm{Hb}$ molecules are more paramagnetic than oxy- $\mathrm{Hb}$ molecules, and the magnetic susceptibility of blood increases linearly with increasing oxygenation. BOLD signal is more sensitive to venous changes than arterial changes because arterial blood is usually fully oxygenated whereas venous blood is more deoxygenated. Functional NIRS not only detects both oxy- and deoxy- $\mathrm{Hb}$ levels, it also detects both arterial and venous blood oxy- and deoxygenation. In contrast, $\mathrm{fMRI}$ detects only deoxygenation in the venous return. Functional NIRS can detect high-resolution temporal signals in msec [1-3] providing real-time monitoring of changes in hemodynamic responses, whereas fMRI usually acquires low-resolution temporal signals when it takes $1 \mathrm{sec}$ to scan the whole brain. 
It is often assumed that relative oxygenation changes with the amount of deoxygenation, but this assumption has not been validated experimentally using fNIRS measurements. There is evidence that BOLD response is shown to reflect pooled local field potential activity [6]. Although neuronal activation and vascular responses are correlated by the "neurovascular coupling" [7], the coupling between oxy- and deoxy-Hb hemodynamic responses have yet to be determined experimentally. It has been shown that transient BOLD signals in fMRI may not necessarily reflect cerebral blood flow (CBF), cerebral metabolic rate of oxygen (CMRO2), or cerebral blood volume (CBV) in simulation model and experimental recording from primary motor area (M1) and supplementary motor area (SMA) [8]. Although there is a high correlation between ASL (arterial spin labeling)-based fMRI, BOLD, and NIRS during an event-related motor activity in human subjects [9], we will show in this study that complex dynamics of temporal coupling and decoupling can occur differentially in the NIRS hemodynamic response. We will use various differential measures of fNIRS hemodynamic signals to quantify the temporal profiles of neurovascular responses (oxygen delivery vs. oxygen extraction, and total blood volume vs. total oxygenation) in real time with respect to the demand of a cognitive task.

A brief review of the computation of the hemodynamic response is provided, applying the modified Beer-Lambert Law [4] to extract the $\mathrm{HbO}_{2}$ and $\mathrm{Hb}$ signals:

$$
I=I_{0} 10^{-\left(\alpha_{\mathrm{Hb}} \Delta c_{\mathrm{Hb}}+\alpha_{\mathrm{Hb}} \Delta c_{\mathrm{HbO}}\right) L} G
$$

and

$$
O D=-\log \frac{I}{I_{0}}=\mu_{a} L D_{P F}+G=\left(\alpha_{\mathrm{Hb}} \Delta c_{\mathrm{Hb}}+\alpha_{\mathrm{HbO}} \Delta c_{\mathrm{HbO}}\right) L
$$

where $O D$ is the optical density of the sample (as determined by the negative log ratio of the detected intensity of light $/$ with respect to the incident intensity of light $I_{o}$ ), $\mu_{a}$ is the absorption coefficient of the tissue, $L$ is the net distance traveled by the light from the light emitter to the NIR sensor, $D_{P F}$ is the differential path-length factor, and $G$ is the geometry factor. $D_{P F}$ accounts for the extra total distance that light travels through the tissue due to scattering. $G$ accounts for light attenuation because of the geometry of the sample. By inducing a small change in $\mu_{a}$ between time $t_{1}$ and $t_{2}$, the change in the optical density will be:

$$
\Delta O D=-\log \left(\mathrm{t}_{1} / \mathrm{t}_{2}\right)=\Delta \mu_{a} L D_{P F}
$$

The total blood volume delivered to the tissue is given by the sum of changes in optical density:

$$
\text { Total Blood Volume }=\Delta c_{\mathrm{HbO}}+\Delta c_{\mathrm{Hb}}
$$

and the amount of oxygenation is given by the difference between changes in optical density: 
Nicoladie D. Tam and George Zouridakis; Temporal decoupling of oxy-and deoxy-hemoglobin hemodynamic responses detected by functional near-infrared spectroscopy (fNIRS), Journal of Biomedical Engineering and Medical Imaging, Volume 1, Issue 2 (2014), PP 18-28

$$
\text { Total Oxygenation }=\Delta c_{\mathrm{HbO}}-\Delta c_{\mathrm{Hb}}
$$

We will denote $\mathrm{HbO}_{2}$ level to represent $\Delta c_{\mathrm{HbO}}$, and $\mathrm{Hb}$ level to represent $\Delta c_{\mathrm{Hb}}$, since the $\Delta c_{\mathrm{HbO}}$ and $\Delta c_{\mathrm{Hb}}$ values are computed as the output optical signals in the optical recording instruments. The total blood volume delivered to the tissue is the sum of $\mathrm{HbO}_{2}$ and $\mathrm{Hb}$ $\left(\mathrm{HbO}_{2}+\mathrm{Hb}\right)$, and the total oxygenation extraction is the difference between $\mathrm{HbO}_{2}$ and $\mathrm{Hb}$ $\left(\mathrm{HbO}_{2}-\mathrm{Hb}\right)$. We will use these two derived measures to characterize the hemodynamic responses, in addition to the convention oxygen delivery $\left(\mathrm{HbO}_{2}\right)$ and oxygen extraction $(\mathrm{Hb})$ measures to quantify the neural activation/deactivation temporal profiles.

We will employ a motor execution task to detect whether there is a temporal decoupling between oxy- and deoxy-Hb temporal hemodynamic responses. It is well known that movement directions can be decoded from the tuning curve representing their preferred movement direction [10-14] using microelectrode recordings of identified neurons in the motor cortex. We will extend this correlation of neural activation with motor execution commands to confirm the hemodynamic response associated with arm movements optically. Since the resultant movement direction can be decoded computationally by the population vector sum of the movement related neurons in a localized region of the cortex electrically [10-14], we will provide the empirical evidence to show that the hemodynamic changes can also be used to decode movement directions optically. We will demonstrate that decoding movement directions requires all four hemodynamic measures of oxygen delivery, extraction, total blood volume and total oxygenation (based on $\mathrm{HbO}_{2}, \mathrm{Hb},\left(\mathrm{HbO}_{2}+\mathrm{Hb}\right)$, and $\left(\mathrm{HbO}_{2}-\mathrm{Hb}\right)$ ), while relying on a single measure of deoxy- $\mathrm{Hb}$ is insufficient to differentiate the movement direction adequately [15-17].

Functional NIRS recordings have the advantage of sampling at a higher temporal resolution (in $\mathrm{KHz}$ ) than $\mathrm{fMRI}$ recordings (in $\mathrm{Hz}$ ), even though most hemodynamic responses occur at a much slower rate than the fNIRS sampling frequency [18]. Nonetheless, the higher temporal resolution of hemodynamic signals is essential for detecting dynamic movement activity, such as the high frequency movements that require maximal effort (ME) [19]. Although previous fNIRS studies had revealed movement related hemodynamic response to motor tasks, most of these studies focused on repetitive motor activation tasks rather than directional arm movements. These studies included finger tapping or oscillator movements [7, 20-24], walking movements [25], movement imagery [26] and activity while solving an anagram task [27]. We will examine how hemodynamic responses in the motor cortex can be used to differentiate the difference between orthogonal arm movement directions by the temporal coupling/decoupling of oxy- and deoxy-Hb signals. 


\section{MATERIALS AND METHODS}

We recorded the optical signals of hemodynamic response in fourteen human subjects while executing a directional arm movement task. Human subjects were asked to execute volitional freehand arm movements in the horizontal $x y$-plane parallel to a desk surface. We included fourteen healthy subjects in this study. All subjects were instructed to initiate rightleft ( $x$-direction) or front-back ( $y$-direction) hand movements between $30-\mathrm{cm}$ targets in a horizontal plane by a sound tone. A 64-channel ISS Imagent ${ }^{\mathrm{TM}}$ optical system is used to record the hemodynamic signals $\left(\mathrm{HbO}_{2}\right.$ and $\left.\mathrm{Hb}\right)$ from both motor cortices bilaterally during the above motor task. We recorded multiple front-back (or right-left) movements (repeating the movement sequence for a period of $5 \mathrm{~min}$ ) so that signal-averaging analysis is used to improve the signal-to-noise ratio. The experimental protocol was approved by the Institutional Review Board.

\section{RESULTS}

Figs. 1 and 2 shows the hemodynamic signals during a backward ( $-y$ direction) arm movement (in a front-to-back direction) $30 \mathrm{~cm}$ in front of the human subject. The graph shows changes in oxy-hemoglobin $\left(\mathrm{HbO}_{2}\right)$ profile (in red) and deoxy-hemoglobin ( $\mathrm{Hb}$ ) profile (in blue), which is correlated temporally with this backward (front-to-back) movement direction. It revealed a continual decrease of oxygen delivery $\left(\mathrm{HbO}_{2}\right)$, but a slight increase in oxygen extraction $(\mathrm{Hb})$ for this arm movement. That is, oxygen delivery and extraction are decoupled temporally with a decrease (deactivation) for oxy- $\mathrm{Hb}$ and a slight increase (activation) for deoxy-Hb. This shows that oxygen delivery decreases while oxygen extraction increases simultaneously.

This result shows that, in contrary to the usual assumption, oxy- $\mathrm{Hb}\left(\mathrm{HbO}_{2}\right)$ and deoxy- $\mathrm{Hb}$ $\mathrm{Hb}$ are always changing in the same direction temporally. That is, oxygen delivery and oxygen extraction do not increase (or decrease) together. Instead, one hemodynamic measure (oxy$\mathrm{Hb}$ ) activates while the other measure (deoxy-Hb) deactivates simultaneously during the same arm movement. This temporal decoupling is revealed by the fNIRS measures (unlike fMRI BOLD signals, which only measures deoxy-Hb level but not oxy-Hb level).

Fig. 2 shows the sum of oxy- $\mathrm{Hb}$ and deoxy- $\mathrm{Hb}\left(\mathrm{HbO}_{2}+\mathrm{Hb}\right)$ (in red) (which represents the total blood volume delivery to the brain tissues in motor cortex), and the difference of oxy-Hb and deoxy- $\mathrm{Hb}\left(\mathrm{HbO}_{2}-\mathrm{Hb}\right)$ (in blue) (which represents total oxygenation in the tissue). These two derived hemodynamics measures reveal that both blood volume and oxygenation decreased continually during the backward arm movement. This suggests a deactivation (decrease) in the total blood delivery and total oxygenation, which are coupled temporally. In contrast, the oxygen delivery (oxy- $\mathrm{Hb}$ ) decreased, while oxygen extraction (deoxy- $\mathrm{Hb}$ ) increased (see Fig. 1), suggesting that oxygen demand was so high that oxygen delivery could not keep up with oxygen extraction. 
Nicoladie D. Tam and George Zouridakis; Temporal decoupling of oxy-and deoxy-hemoglobin hemodynamic responses detected by functional near-infrared spectroscopy (fNIRS), Journal of Biomedical Engineering and Medical Imaging, Volume 1, Issue 2 (2014), PP 18-28

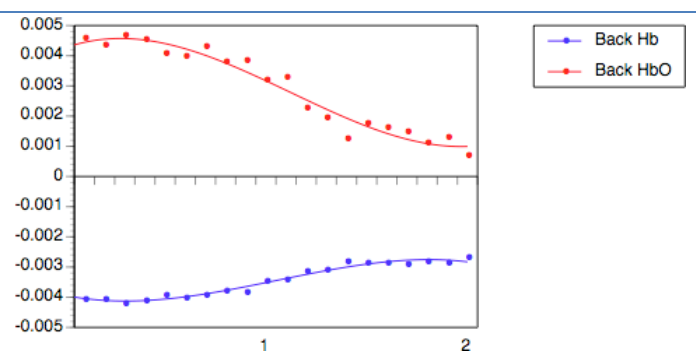

Figure 1: Oxy- and deoxy-Hb hemodynamic responses of arm movement toward backward (frontto-back) in $-y$ direction. Sound tone is used to instruct movement onset is at time $0 .\left[\mathrm{HbO}_{2}\right.$ signal is shown in red, and $\mathrm{Hb}$ in blue. $X$-axis is in sec, $y$-axis is in decoded optical intensity units.]

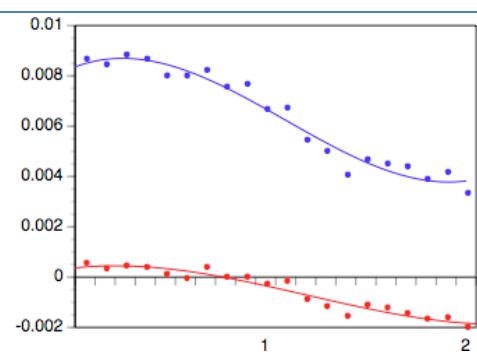

$\rightarrow$ Back $(\mathrm{HbO}-\mathrm{Hb})$ $\rightarrow$ Back $(\mathrm{HbO}+\mathrm{Hb})$

Figure 2: Hemodynamic responses as measured by the sum of $\mathrm{HbO}_{2}$ and $\mathrm{Hb}\left(\mathrm{HbO}_{2}+\mathrm{Hb}\right)$, and difference of $\mathrm{HbO}_{2}$ and $\mathrm{Hb}\left(\mathrm{HbO}_{2}-\mathrm{Hb}\right)$ for the same backward arm movement shown in Fig. 1. [( $\left.\mathrm{HbO}_{2}+\mathrm{Hb}\right)$ signal is shown in red, $\left(\mathrm{HbO}_{2}-\mathrm{Hb}\right)$ signal is shown in blue.]

This analysis shows that three of the hemodynamic variables - oxygen delivery $\left(\mathrm{HbO}_{2}\right)$, total blood volume $\left(\mathrm{HbO}_{2}+\mathrm{Hb}\right)$, and total oxygenation $\left(\mathrm{HbO}_{2}-\mathrm{Hb}\right)$ - all decreased in the backward movement direction (front-to-back direction), while oxygen extraction ( $\mathrm{Hb}$ ) level increased slightly. This shows a temporally decoupled response of oxygen extraction from the above three hemodynamic variables. Thus, the optical recordings revealed that not all four hemodynamic measures $\left(\mathrm{HbO}_{2}, \mathrm{Hb},\left(\mathrm{HbO}_{2}+\mathrm{Hb}\right)\right.$, and $\left.\left(\mathrm{HbO}_{2}-\mathrm{Hb}\right)\right)$ are coupled temporally (or activated simultaneously) in a task related response.

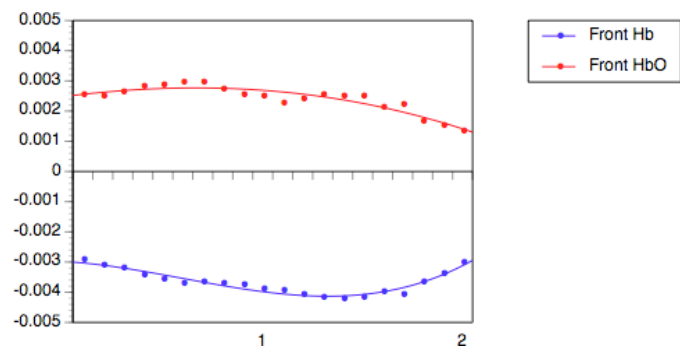

Figure 3: Oxy- and deoxy-Hb hemodynamic responses of arm movement toward frontward direction (back-to-front) in $+y$ direction.

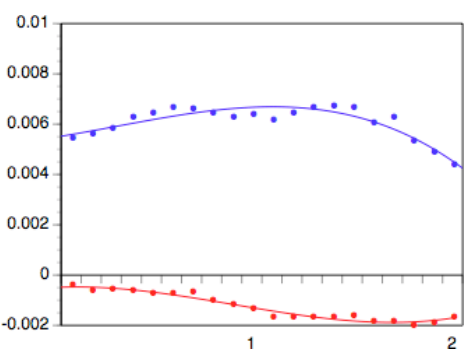

- Front (HbO-Hb) $\rightarrow$ Front $(\mathrm{HbO}+\mathrm{Hb})$

Figure 4: Hemodynamic responses as measured by the sum of $\mathrm{HbO}_{2}$ and $\mathrm{Hb}\left(\mathrm{HbO}_{2}+\mathrm{Hb}\right)$, and difference of $\mathrm{HbO}_{2}$ and $\mathrm{Hb}\left(\mathrm{HbO}_{2}-\mathrm{Hb}\right)$ for the same frontward arm movement shown in Fig. 3.

In contrast, frontward (back-to-front) arm movement in $+y$ direction (Fig. 3) shows an opposite change in the hemodynamic response. It shows a slight increase (then a decrease) in the oxy-Hb level, but an opposite temporal profile (a slight decrease and then an increase) in deoxy-Hb level. That is, a temporal decoupling between oxygen delivery and oxygen extraction occurred for the frontward movement. But for the derived measures of total blood volume and oxygenation (Fig. 4), i.e., $\left(\mathrm{HbO}_{2}+\mathrm{Hb}\right)$ and $\left(\mathrm{HbO}_{2}-\mathrm{Hb}\right)$, they showed opposite changes temporally, unlike the simultaneous deactivation for backward arm movements. This shows that the same motor cortical area was activated/deactivated differently for both frontward and backward arm movements as revealed temporally by the opposite changes 
(increasing/decreasing) in oxygen delivery, extraction, total oxygenation and total blood volume (by comparing Figs. 1-4).

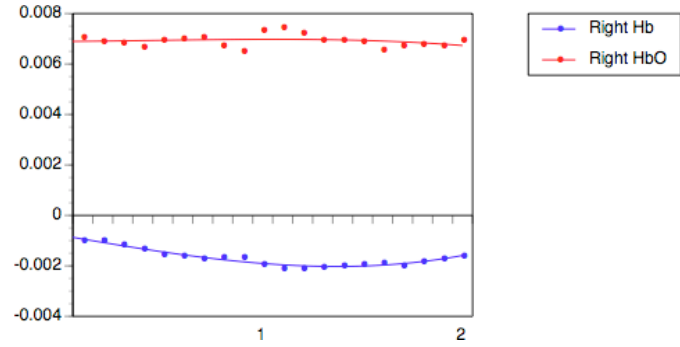

Figure 5: Oxy- and deoxy-Hb hemodynamic responses of arm movement toward rightward direction (right-to-left) in $+x$ direction.

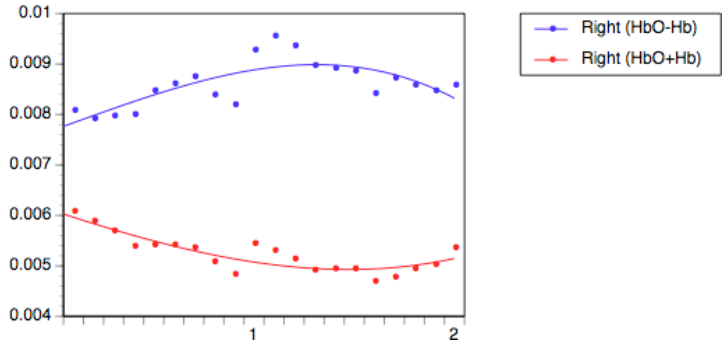

Figure 6: Hemodynamic responses as measured by the sum of $\mathrm{HbO}_{2}$ and $\mathrm{Hb}\left(\mathrm{HbO}_{2}+\mathrm{Hb}\right)$, and difference of $\mathrm{HbO}_{2}$ and $\mathrm{Hb}\left(\mathrm{HbO}_{2}-\mathrm{Hb}\right)$ for the same rightward arm movement shown in Fig. 5.

In order to illustrate the differences in temporal hemodynamic profiles between front-back and right-left movements, Figs. 5-8 show the hemodynamic responses for the right and left movements (compared to Figs. 1-4 for the front and back movements recorded from the same optrode in the same human subject). Fig. 5 shows the temporal decoupling response with no change in oxy- $\mathrm{Hb}$, but a slight decrease in deoxy-Hb level for the rightward ( $+x$ direction) arm movement. But for the derived measures of total blood volume and oxygenation (Fig. 6), i.e., $\left(\mathrm{HbO}_{2}+\mathrm{Hb}\right)$ and $\left(\mathrm{HbO}_{2}-\mathrm{Hb}\right)$, they showed opposite changes temporally. They are decoupled temporally.

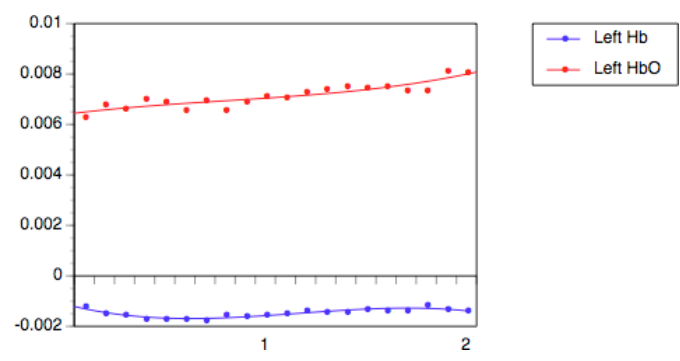

Figure 7: Oxy- and deoxy-Hb hemodynamic responses of arm movement toward leftward direction (left-to-right) in $-x$ direction.

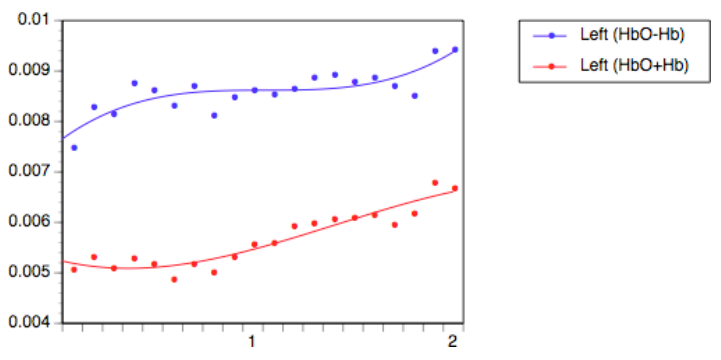

Figure 8: Hemodynamic responses as measured by the sum of $\mathrm{HbO}_{2}$ and $\mathrm{Hb}\left(\mathrm{HbO}_{2}+\mathrm{Hb}\right)$, and difference of $\mathrm{HbO}_{2}$ and $\mathrm{Hb}\left(\mathrm{HbO}_{2}-\mathrm{Hb}\right)$ for the same leftward arm movement shown in Fig. 7.

For leftward ( $-x$ direction) arm movements, Fig. 7 shows there is a slight increase in oxy-Hb with no change in deoxy- $\mathrm{Hb}$ level. If oxy- $\mathrm{Hb}$ and deoxy- $\mathrm{Hb}$ levels were to use as an indicator to differentiate between right and left arm movements by comparing Fig. 5 and Fig. 7, then activation of oxy-Hb suggests leftward movement while deactivation of deoxy-Hb suggests rightward movement. But if the derived measures of total blood volume and oxygenation were used to compare the difference, i.e., $\left(\mathrm{HbO}_{2}+\mathrm{Hb}\right)$ and $\left(\mathrm{HbO}_{2}-\mathrm{Hb}\right)$, then the temporal profile for the above two measures are decoupled for rightward movement (see Fig. 6) but coupled for leftward movement (see Fig. 8). 
Nicoladie D. Tam and George Zouridakis; Temporal decoupling of oxy- and deoxy-hemoglobin hemodynamic responses detected by functional near-infrared spectroscopy (fNIRS), Journal of Biomedical Engineering and Medical Imaging, Volume 1, Issue 2 (2014), PP 18-28

Thus, the activation/deactivation patterns vary depending on which temporal hemodynamic profile is used as the criterion to characterize the neural response. The temporal changes in hemodynamic response for the neural population under the same optrode vary depending on whether the arm movement is right, left, front or back (i.e., $+x,-x,+y$ or $-y$ directions). They can be temporally coupled (or temporally decoupled) in any combination among the four hemodynamic measures $\left[\mathrm{HbO}_{2}, \mathrm{Hb},\left(\mathrm{HbO}_{2}+\mathrm{Hb}\right)\right.$ and $\left.\left(\mathrm{HbO}_{2}-\mathrm{Hb}\right)\right]$ we examined in this study.

\section{DISCUSSION}

This study demonstrated empirically that oxygen delivery and extraction can be coupled (and decoupled) temporally by the same neural tissue on demands, depending on the specific movement execution directions. This dynamical change in temporal profiles shows the complexity (as well as the unique differential changes) in oxy- $\mathrm{Hb}$ and deoxy- $\mathrm{Hb}$ signals associated with a specific movement direction. Analysis of the fNIRS signals representing the hemodynamic responses demonstrated that different measures of hemodynamic responses showed differential changes temporally to the same motor task. Specifically, the differential activation and deactivation of hemodynamic responses cannot be assumed to occur simultaneously without actual measurements of the oxy-Hb and deoxy-Hb signals.

The analysis revealed that various measures of hemodynamic vascular responses exhibit decoupled responses temporally during the same movement direction, while the hemodynamic responses are not always opposite for the opposite movement direction. Oxygen delivery, extraction, total blood volume, and total oxygenation all can be coupled/decoupled temporally in the same movement direction, but partially decoupled/coupled in opposite movement direction. The temporal decoupling may not occur in opposite manner (i.e., activation vs. deactivation) for the opposite movement direction. Similar temporal decouplings were also reported $[16,17]$.

The decoupling is likely due to a transient supply of oxygen not keeping up with the oxygen demand in the neural tissue. Although oxygen consumption increases with metabolic activity, it can be due to increased metabolism in supportive cells, such as glial cells that regulate ionic concentration and neurotransmitter metabolism. Hemodynamic response is known to be associated with oxygen demand and metabolic activity, but may not necessarily coupled with neuronal activity directly [28]. Since we did not record both electrical and optical signals simultaneously, it is unknown in this study whether hemodynamic vascular response is related temporally to the electrical firing activities or synaptic events.

Most importantly, the differential changes in the temporal hemodynamic responses revealed that oxygen demands can be accomplished by two independent processes: oxygen extraction and oxygen delivery. Total oxygen delivery can be maximized by an increase in total blood volume delivered $\left(\mathrm{HbO}_{2}+\mathrm{Hb}\right)$, and by an increase in partial pressure of oxygen $\left(\mathrm{P}_{\mathrm{O}_{2}}\right)$ 
saturation in the hemoglobin molecules. Oxygen extraction is accomplished by unloading oxygen molecules from hemoglobin molecules, which is detected by a decrease in $\mathrm{P}_{\mathrm{O} 2}$ in the hemodynamic signal.

The decoupled temporal dynamics is a phenomenon often observed during physical exercise in the peripheral system (during high muscle oxygen demand), when oxygen consumption is so high that oxygen extraction exceeds the ability for oxygen to be delivered to the target tissue by blood. It is only when oxygen demand is not intense that oxygen delivery is sufficient to meet the demand by the total blood volume delivered under normal non-hypoxic circumstances. This phenomenon is observed during non-demanding physical exercise with $\mathrm{P}_{\mathrm{O} 2}$ maintaining at 96-99\% saturation normally. Hypoxic condition can occur during strenuous exercise when metabolic demand is so high that $\mathrm{P}_{\mathrm{O} 2}$ saturation in hemoglobin molecules will drop below $96 \%$, creating the temporally decoupled hemodynamic phenomenon.

It can be deduced that, when neural metabolic demand is so high, micro-hypoxic condition can occur locally at the activation site. It is only during normal (non-hypoxic) condition that, without exceeding this limit, the oxygen delivery can keep up with extraction. This could account for the above differential temporal decoupled phenomenon in the hemodynamic responses illustrated earlier.

This interpretation is consistent with the complex transient biphasic temporal cerebral oxygenation responses in response to intense oscillatory motor stimulation [21]. It is also consistent with similar findings of regional cerebral blood oxygenation ( $\mathrm{CCBO}$ ) in the motor cortex and blood flow velocity changes (CBFV) in the middle cerebral artery in a combined NIRS and transcranial Doppler Sonography (TCD) study for sequential finger opposition task [23]. The size of hemodynamic response also reported [22] to be dependent on the resting period between sequential motor executions, which peaked maximally with a 30 -sec resting period in finger tapping task.

These findings are consistent with our findings that the hemodynamic responses are complex dynamical interactions among many various vascular components to produce the temporal changes $[16,17]$. This shows that oxygen delivery and extraction can change independently and may decouple temporally. So if the neuronal activation/deactivation response is characterized by only one of these hemodynamic measures (either oxy- $\mathrm{Hb}$ or deoxy- $\mathrm{Hb}$ ) without the other, it will miss the complete description of the temporal response that represents both oxygen delivery and extraction dynamics.

Most importantly, if only deoxy-Hb level were used to infer the hemodynamic response as neural activation (as in fMRI BOLD signal analysis), it could mischaracterize the neural response since fNIRS signals can reveal a simultaneous increase in oxy- $\mathrm{Hb}$ level but a decrease in deoxy$\mathrm{Hb}$ level (or vice versa) during the same motor task. Thus, no single measure of the hemodynamic response is sufficient to characterize the neural activation/deactivation pattern temporally. Since fNIRS recordings can detect a much higher temporal resolution (in msec) 
Nicoladie D. Tam and George Zouridakis; Temporal decoupling of oxy- and deoxy-hemoglobin hemodynamic responses detected by functional near-infrared spectroscopy (fNIRS), Journal of Biomedical Engineering and Medical Imaging, Volume 1, Issue 2 (2014), PP 18-28

than fMRI (in sec), the hemodynamic response can change so rapidly that it can increase and then decrease within the time frame of less than $1 \mathrm{sec}$ (which is less than the typical scanning time required for $\mathrm{fMRI}$ recordings). It remains to be confirmed with simultaneous recordings of both $\mathrm{fNIRS}$ and $\mathrm{fMRI}$ in subsequent studies to determine whether any direct evidence will support the above findings experimentally.

\section{CONCLUSION}

This study demonstrated the experimental evidence that differential temporal changes in hemodynamic measures of $\mathrm{HbO}_{2}, \mathrm{Hb},\left(\mathrm{HbO}_{2}+\mathrm{Hb}\right)$ and $\left(\mathrm{HbO}_{2}-\mathrm{Hb}\right)$ can occur in NIRS signals during the same motor task. The increase/decrease in the above hemodynamic measures can be decoupled from each other temporally. The temporal changes in hemodynamic response are not necessarily opposite for arm movements that were made in the opposite direction. If neural activation were deduced based on monitoring the deoxy- $\mathrm{Hb}$ level in the BOLD signals alone without monitoring the oxy- $\mathrm{Hb}$ level, it may represent a partial record of the hemodynamic activities compared to the more complete record of both oxy- and deoxy-Hb signals recorded by the fNIRS signals.

\section{ACKNOWLEDGEMENT}

We would like to thank Ms. Krista Smith for the helpful suggestions and for proofreading the manuscript.

\section{REFERENCES}

1. Calderon-Arnulphi, M., A. Alaraj, and K. Slavin, Near infrared technology in neuroscience: past, present and future. Neurol Res, 2009. 31(6): p. 605 - 614.

2. Hoshi, Y., Towards the next generation of near-infrared spectroscopy. Philos Trans A Math Phys Eng Sci, 2011. 369(1955): p. 4425-39.

3. Pellicer, A. and C. Bravo Mdel, Near-infrared spectroscopy: a methodology-focused review. Semin Fetal Neonatal Med, 2011. 16(1): p. 42-9.

4. Cope, M., et al., Methods of quantitating cerebral near infrared spectroscopy data. Adv Exp Med Biol, 1988. 222: p. 183-9.

5. Montcel, B., R. Chabrier, and P. Poulet, Time-resolved absorption and hemoglobin concentration difference maps: a method to retrieve depth-related information on cerebral hemodynamics. Opt Express, 2006. 14(25): p. 12271-87.

6. Logothetis, N.K., et al., Neurophysiological investigation of the basis of the fMRI signal. Nature, 2001. 412(6843): p. 150-7.

7. Toronov, V., et al., Investigation of human brain hemodynamics by simultaneous near-infrared spectroscopy and functional magnetic resonance imaging. Med Phys, 2001. 28(4): p. 521-7.

8. Obata, T., et al., Discrepancies between BOLD and flow dynamics in primary and supplementary motor areas: application of the balloon model to the interpretation of BOLD transients. Neuroimage, 2004. 21(1): p. 14453. 
Journal of Biomedical Engineering and Medical Imaging, Volume 1, Issue 2, April 2014

9. Huppert, T.J., et al., A temporal comparison of BOLD, ASL, and NIRS hemodynamic responses to motor stimuli in adult humans. Neuroimage, 2006. 29(2): p. 368-82.

10. Schwartz, A.B., Direct cortical representation of drawing. Science, 1994. 265(5171): p. 540-2.

11. Kettner, R.E., A.B. Schwartz, and A.P. Georgopoulos, Primate motor cortex and free arm movements to visual targets in three-dimensional space. III. Positional gradients and population coding of movement direction from various movement origins. J Neurosci, 1988. 8(8): p. 2938-47.

12. Georgopoulos, A.P., R.E. Kettner, and A.B. Schwartz, Primate motor cortex and free arm movements to visual targets in three-dimensional space. II. Coding of the direction of movement by a neuronal population. J Neurosci, 1988. 8(8): p. 2928-37.

13. Georgopoulos, A.P., A.B. Schwartz, and R.E. Kettner, Neuronal population coding of movement direction. Science, 1986. 233(4771): p. 1416-9.

14. Lin, S., J. Si, and A.B. Schwartz, Self-organization of firing activities in monkey's motor cortex: trajectory computation from spike signals. Neural Comput, 1997. 9(3): p. 607-21.

15. Tam, N.D. and G. Zouridakis, Optical imaging of motor cortical activation using functional near-infrared spectroscopy. BMC Neuroscience, 2012. 13(Suppl 1): p. P27.

16. Tam, N.D. and G. Zouridakis, Optical imaging of motor cortical hemodynamic response to directional arm movements using near-infrared spectroscopy. American Journal of Biomedical Engineering, 2013. 3(2): p. 1117.

17. Tam, N.D.Z., George, Decoding Movement Direction from Motor Cortex Recordings Using Near-Infrared Spectroscopy.. (in press). Infrared Spectroscopy: Theory, Developments and Applications2014, Hauppauge, NY: Nova Science Publishers, Inc.

18. Pouliot, P., et al., Nonlinear hemodynamic responses in human epilepsy: a multimodal analysis with fNIRSEEG and fMRI-EEG. J Neurosci Methods, 2012. 204(2): p. 326-40.

19. Colier, W.N.J.M., et al., Detailed evidence of cerebral hemoglobin oxygenation changes in response to motor activation revealed by a continuous wave spectrophotometer with $10 \mathrm{~Hz}$ temporal resolution. Proc SPIE, 1997. 2979: p. 390-396.

20. Kuboyama, N., et al., The effect of maximal finger tapping on cerebral activation. J Physiol Anthropol Appl Human Sci, 2004. 23(4): p. 105-10.

21. Obrig, H., et al., Cerebral oxygenation changes in response to motor stimulation. J Appl Physiol, 1996. 81(3): p. 1174-83.

22. Obrig, $\mathrm{H}$., et al., Length of resting period between stimulation cycles modulates hemodynamic response to a motor stimulus. Adv Exp Med Biol, 1997. 411: p. 471-80.

23. Hirth, C., et al., Simultaneous assessment of cerebral oxygenation and hemodynamics during a motor task. A combined near infrared and transcranial Doppler sonography study. Adv Exp Med Biol, 1997. 411: p. 461-9.

24. Leff, D.R., et al., Assessment of the cerebral cortex during motor task behaviours in adults: a systematic review of functional near infrared spectroscopy (fNIRS) studies. Neuroimage, 2011. 54(4): p. 2922-36.

25. Suzuki, M., et al., Prefrontal and premotor cortices are involved in adapting walking and running speed on the treadmill: an optical imaging study. Neuroimage, 2004. 23(3): p. 1020-6.

26. Wriessnegger, S.C., J. Kurzmann, and C. Neuper, Spatio-temporal differences in brain oxygenation between movement execution and imagery: a multichannel near-infrared spectroscopy study. Int J Psychophysiol, 2008. 67(1): p. 54-63.

27. Tachtsidis, l., et al., Investigation of frontal cortex, motor cortex and systemic haemodynamic changes during anagram solving. Adv Exp Med Biol, 2008. 614: p. 21-8.

28. Biswal, B.B., et al., Decoupling of the hemodynamic and activation-induced delays in functional magnetic resonance imaging. J Comput Assist Tomogr, 2003. 27(2): p. 219-25. 